\title{
Dysfunction in Automatic Processing of Emotional Facial Expressions in Patients with Obstructive Sleep Apnea Syndrome: An Event-Related Potential Study
}

This article was published in the following Dove Press journal:

Nature and Science of Sleep

\author{
Renjun Lv $\mathbb{1}^{1-5}$ \\ Shanjing $\mathrm{Nie} \mathbb{D}^{1-4}$ \\ Zhenhua Liu ${ }^{6}$ \\ Yunliang Guo'-4 \\ Yue Zhang ${ }^{7}$ \\ Song $\mathrm{Xu}^{1-4}$ \\ Xunyao Hou ${ }^{1-4}$ \\ Jian Chen ${ }^{1-4}$ \\ Yingjuan $\mathrm{Ma}^{8}$ \\ Zhongyu Fan $\mathbb{D}^{1-5}$ \\ Xueping Liu $^{1-4}$
}

'Department of Geriatrics, Shandong Provincial Hospital Affiliated to Shandong First Medical University, Jinan 25002I, Shandong,

People's Republic of China; 'Department of

Geriatric Neurology, Shandong Provincial

Hospital Affiliated to Shandong First Medical

University, Jinan 25002I, Shandong, People's

Republic of China; ${ }^{3}$ Anti-Aging Monitoring

Laboratory, Shandong Provincial Hospital

Affiliated to Shandong First Medical University,

Jinan 25002I, Shandong, People's Republic of

China; ${ }^{4}$ Department of Anti-Aging, Shandong

Provincial Hospital Affiliated to Shandong First

Medical University, Jinan 25002I, Shandong,

People's Republic of China; ${ }^{5}$ Shandong

Provincial Hospital, Shandong First Medical

University \& Shandong Academy of Medical

Sciences, Jinan 25002I, Shandong, People's

Republic of China; ${ }^{6}$ Center of Sleep Medicine,

Shandong Provincial Hospital Affiliated to

Shandong First Medical University, Jinan

25002I, Shandong, People's Republic of China:

${ }^{7}$ Department of Geriatric Neurology,

Shandong Provincial Hospital Affiliated to

Shandong University, Jinan 25002I, Shandong,

People's Republic of China; ${ }^{8}$ Department of

Otolaryngology Head and Neck Surgery,

Shandong Provincial Hospital Affiliated to

Shandong First Medical University, Jinan,

Shandong, 25002I, People's Republic of China

Correspondence: Xueping Liu

Tel +86-53168777I65

Email Liuxueping1962@163.com

\begin{abstract}
Aim: Obstructive sleep apnea syndrome (OSAS) is a prevalent chronic disease characterized by sleep fragmentation and intermittent hypoxemia. Several studies suggested that electrophysiological changes and neurocognitive abnormalities occurred in OSAS patients. In this study, we compared automatic processing of emotional facial expressions schematic in OSAS patients and matched healthy controls via assessing expression-related mismatch negativity (EMMN).

Methods: Twenty-two OSAS patients (mean age 44.59 years) and twenty-one healthy controls (mean age 42.71 years) were enrolled in this study. All participants underwent Montreal Cognitive Assessment (MoCA) scale test and polysomnographic recording. An expressionrelated oddball paradigm was used to elicit EMMN and the electroencephalogram was recorded and analyzed. Furthermore, Pearson's correlations were calculated to discuss the correlation between neuropsychological test scores, clinical variables and electrophysiological data.

Results: Compared with healthy controls, OSAS sufferers demonstrated significantly reduced EMMN mean amplitudes within corresponding time intervals, regardless of happy or sad conditions. Meanwhile, we observed that amplitude of sad EMMN was larger (more negative) than happy EMNN in healthy controls, while not in patients. Moderate correlations were found between MoCA test scores, sleep parameters and EMMN amplitudes.

Conclusion: Our findings suggested pre-attentive dysfunction of processing emotional facial expressions in patients with OSAS, without the existence of negative bias effect. Moreover, correlation analysis showed that clinical characteristics of OSAS patients could affect EMMN amplitudes. Further studies on the advantages of EMMN as clinical and electrophysiological indicators of OSAS are warranted.
\end{abstract}

Keywords: OSAS, EMMN, facial expressions, pre-attentive processing, negative bias effect

\section{Introduction}

Obstructive sleep apnea syndrome (OSAS) is a common sleep-related breathing disorder due to upper airway obstruction that results in apnea or hypopnea, which further leads to hypercapnia, sleep fragmentation and intermittent hypoxemia. ${ }^{1,2}$ Such disorders with sleep alterations are regarded as the underlying causes of cognitive dysfunction in OSAS. ${ }^{3}$ Individuals with OSAS often feel unrested, fatigued, and sleepy during the day, which may be due to hypoxemia and sleep fragmentation. ${ }^{4}$ Recent international study indicates that the estimated prevalence of OSAS is $14 \%$ in men and $5 \%$ in 
women. ${ }^{5}$ A research suggests that OSAS may promote the development of neurodegenerative disease. ${ }^{6}$ Many studies have reported many domains of neurocognitive impairments in patients with OSAS, including selective attention, ${ }^{7}$ information processing speed, ${ }^{8}$ working memory. ${ }^{9}$

Emotion processing is an advanced cognitive process that plays an important role in interpersonal communication. In addition, facial expressions are important ecological stimulus (emotional stimulus) as they convey the most fundamental information such as an individual's mood and intention in social interactions, promoting interpersonal communication and accurate and integrated perception of emotional information is vital for effective social communication. Clinical evidence suggests that OSAS can cause mood regulation disorders, leading to aggressive behavior, depressive and anxiety disorders. ${ }^{10}$ While to our knowledge, direct research on socio-emotional processing in OSAS is not available at present, which demands further elucidation.

Event-related potential (ERP) is a non-invasive and relatively objective measurement that reflects cognitive processing, which has been increasingly used as a biological marker in a range of psychiatric and neurological conditions, ${ }^{11}$ as well as OSAS. ${ }^{12}$ Mismatch negativity $(\mathrm{MMN})$ is an endogenous ERP component and serves as an index for the pre-attentive processing of the detected changes in environmental information. ${ }^{13}$ It could reflect the difference between the ERP elicited by deviant (infrequent) and standard (frequent) stimuli. ${ }^{14}$ The auditory modality of MMN has been well defined, which was studied in chronic intermittent nocturnal hypoxemia conditions in OSAS, and found that the auditory MNN latency prolonged progressively, as hypoxemia levels aggravated, which usually indicates impaired automatic auditory processing. ${ }^{15}$ Another study found that OSAS patients showed significantly prolonged latency of auditory $\mathrm{MMN}$, this result suggests that the brain function of automatic processing is impaired. ${ }^{16}$ Visual MMN (vMMN) response is the visual counterpart of the auditory MMN, ${ }^{17}$ and convincing evidence has been provided for the fact that vMMN exists objectively, which could be obtained not only with physical stimuli, for instance, motion direction, ${ }^{18}$ colors, ${ }^{19}$ spatial frequency, ${ }^{20}$ but also with biological stimuli, such as emotional expressions. ${ }^{21}$

The phenomenon that biological stimuli could elicit vMMN has been verified. Using frequent happy faces (standard stimuli) and infrequent neutral faces (deviant stimuli) in an oddball paradigm, Susac et al observed an emotion-related negativity, an effect similar to auditory $\mathrm{MMN} .^{22}$ In another study, researchers have found expression-related vMMN (called expression MMN, EMMN) by using an oddball paradigm, with neutral faces served as standard stimuli and happy and sad faces served as deviant stimuli. ${ }^{23}$ Similarly, Astikainen and Hietanen have obtained more reliable EMMN by the usage of pictures with different facial features. ${ }^{24}$ Studies have shown that schematic faces used in oddball paradigm are useful and reliable for studying the brain response to emotional faces, which indicates that the task-irrelevant schematic facial expressions could elicit EMMN. ${ }^{23,25}$ A study indicates that EMMN is a biomarker that can measure cognitive bias in pre-attentive processing in remitted late-life depression. ${ }^{26}$ EMMN reflects automatic and non-conscious facial expression information processing, and has been increasingly applied in various psychological and neurological diseases, such as schizophrenia, ${ }^{27}$ primary insomnia, ${ }^{28}$ migraine. $^{29}$

To our knowledge, there is no study investigating automatic changes in processing facial expressions of OSAS patients via the EMMN component. Thus, in the present study, we measured EMMN elicited by schematic facial expressions (neutral, happy and sad) with an expressionrelated oddball paradigm and explored correlations between EMMN amplitudes and clinical characteristics. This study is the first to investigate the characteristics of expression-related vMMN in patients with OSAS to further understand the neurocognitive information of expression processing at the pre-attentive stage. We hypothesized that OSAS patients suffered from cognitive impairment of facial expression at the pre-attentive stage.

\section{Materials and Methods}

\section{Subjects}

Twenty-nine OSAS patients (17 males) were recruited from both the outpatient and inpatient units in the Department of Neurology at Shandong Provincial Hospital Affiliated to Shandong First Medical University. We also recruited twenty-six healthy controls (HC) (14 males) from hospital/laboratory staff, which were matched with the OSAS group, in terms of age, gender, and education level. All OSAS patients and 26 healthy controls completed a full-night of polysomnographic (PSG) evaluation to diagnose or exclude OSAS.

The diagnosis of OSAS (apnea hypopnea index (AHI) >5) was based on the Clinical Practice Guideline for Diagnostic Testing for Adult Obstructive Sleep Apnea: An American Academy of Sleep Medicine Clinical Practice 
Guideline. ${ }^{30}$ Inclusion criteria for the OSAS group were: patients diagnosed with OSAS according to the above guideline; educational level with either high school education or higher level of education; had no history of treatment for OSAS and exhibited no visual impairment (had normal or corrected-to-normal vision) and right-handed. The exclusion criteria included factors that may cause cognitive impairment and affect sleep or daytime sleepiness, including other sleep disorders, dementia, cerebrovascular disease, history of brain traumatic injury, psychiatric disorders (ie, depression, psychosis, and anxiety disorders), chronic respiratory disease, alcohol/drug abuse such as antidepressants, hypnotics.

In addition, 7 OSAS patients (3 males) were excluded three patients for drift artefacts and excessive blink interference within electroencephalogram (EEG) data, four remaining for incomplete or lost clinical data. Finally, we included 22 OSAS patients (17 males). As for all healthy controls (normal: AHI < 5), five participants ( 2 males) had to be excluded. Three for technical problems during recording and two for excessive blink interference. Thus, total of 21 healthy controls (11 males) were included for further analysis.

The ethical committee of the Shandong Provincial Hospital Affiliated to Shandong First Medical University (Approval No. 2018-215) approved the study, which was in accordance with the ethical principles of the Declaration of Helsinki. All participants provided written informed consent.

\section{PSG Monitoring}

All subjects were assessed for PSG ( Compumedics Grael Acquisition System) recordings for at least 7 hours in Center of Sleep Medicine at Shandong Provincial Hospital Affiliated to Shandong First Medical University. Lowest oxygen saturation during the night ( $\mathrm{MmSpO} \%)$ and mean oxygen saturation during the night (MSpO2) were measured by a transcutaneous finger pulse oximeter. Sleep parameters were evaluated according to American Academy of Sleep Medicine Clinical Practice Guidelines, and AHI, MmSpO2\% and $\mathrm{MSpO}$, arousal index (AI), and oxygen desaturation index (ODI) were analyzed by experienced technicians.

\section{Evaluation of Neurocognitive Impairment and Daytime Sleepiness of OSAS}

Montreal Cognitive Assessment (MoCA) scale is used to screen mild cognitive impairment quickly and conveniently for all participants. ${ }^{31}$ It takes about 15 minutes to perform the MoCA test, with a score of 30 points, which is used to assess eight aspects of cognitive functions, including
Visuospatial \&Executive, Naming, Memory\&delayed recall, Attention, Language, Abstraction, Orientation. Scores below 26 are considered neurocognitive impairment.

The Epworth Sleepiness Scale (ESS) ${ }^{32}$ is a short, easy to administer, and self-rated questionnaire that includes eight items concerning everyday situations, and is used to evaluate daytime sleepiness for all participants. Responses to each item are ranked from 0 to 3 according to the chance of dozing $(0=$ no chance of dozing, $1=$ slight chance of dozing, $2=$ moderate chance of dozing, $3=$ high chance of dozing). A total score $\geq 10$ indicates the presence of sleepiness.

To ensure the reliability and accuracy of measurement, subjects were required to perform these tests between 10:00 and 11:00 a.m., and were evaluated by the same professional psychologist.

\section{Stimuli and Procedures}

Fifty-four schematic faces with happy, sad, and neutral expressions were presented at the center of the screen (23 in.) and each schematic face were made up of 18 different facial models, which were modulated by changing the shape and distance of the facial features in order to avoid low-level processing of facial emotion. Each stimulus lasted for $150 \mathrm{~ms}$, with an inter-stimulus interval (offset-to-onset) of $450 \mathrm{~ms}$ and a visual angle of $3.8^{\circ} \times 4.0^{\circ}$ at a $70 \mathrm{~cm}$ viewing distance (Figure 1).

The stimulus procedure of expression-related oddball paradigm (Figure 2): neutral faces as standard stimuli, happy and sad faces as deviant stimuli. The target stimuli, fixation crosses with enlarging sizes, were always shown at the center of the screen, which prevented contamination by artifacts associated with motor response and active tasks. The changes in the size of the fixation cross were independent of the presentation of different facial expressions (neutral, happy and sad), and participants were instructed that emotional faces on two sides of screen did not relate to their performance on the task. Three sequences were conducted with 211 trials for each (standard: 151 neutral faces; deviant: 30 happy and 30 sad faces). To establish a sensory memory pattern, 10 standard stimuli (neutral faces) were shown at the beginning of the stimulus sequence, and there were at least two standards between successive deviants. The participants were seated in a quiet and electrical shielding room between 10:00 and 11:00 a.m., and were asked to pay attention to unpredictable changes in the cross ("+") at the center of the screen and respond by pressing the button ("/") on the keyboard as rapidly as possible, while ignoring facial stimuli on both 
sides of the cross. Three short exercises were conducted before the formal test to familiarize the subjects with the procedure, but their EEG was not recorded.

\section{Recording of EEG Signals}

EEG were continuously recorded using the Neurolab EEG/ ERPs 32-channel Amplifier system with $\mathrm{Ag}-\mathrm{AgCl}$ active electrodes. The EEG recording electrodes were placed according to the international 10-20 system, and the reference electrode was placed on Fpz, two electrodes are placed above and below the right eye and $10 \mathrm{~mm}$ from the right outer canthi to record the vertical and horizontal electrooculogram (EOG) signals. Electrode impedance was always held below $5 \mathrm{k} \Omega$ throughout the experiment, hardware high pass filter $0.05 \mathrm{~Hz}$ and the low pass filter of $100 \mathrm{~Hz}$ and the sampling rate of $1000 \mathrm{~Hz}$ was used.

\section{EEG Data Analysis and Measurement}

The EEG data were analyzed by ASA 4.9.3 software, and independent component analysis (ICA) was applied to correct EOG artifacts. ${ }^{33}$ After correction for EOG artifacts, EEG with a $600 \mathrm{~ms}$ period was segmented into the epoch from $100 \mathrm{~ms}$ pre-stimulus to $500 \mathrm{~ms}$ post-stimulus. The trials contaminated with artifacts exceeding $\pm 100 \mu \mathrm{V}$ and the target at which the participants' responses occurred were excluded before averaging. The EEG segments were averaged for standard and deviant stimuli and singlesubject waveforms were used to form group-averaged waveforms.

As showed in Figure 3, all emotion stimuli elicited P1, N170 and P250 components in OSAS patients and healthy groups. EMMN is obtained by subtracting ERP of the standard stimuli (neutral faces) from the ERP of the deviant stimuli (happy and sad faces), respectively. The mean amplitudes of EMMN in two time windows were measured, which included $150-250 \mathrm{~ms}$ time window after stimulus onset as early EMMN, and $250-350 \mathrm{~ms}$ time window after stimulus onset as late EMMN. Since the reported posterior distributions ${ }^{20}$ and limitation of the 32site montage, only subsets of the sites were further analyzed for EMMN. This included lateral sites P7 and $\mathrm{P} 8$, centered sites $\mathrm{O} 1$ and $\mathrm{O} 2$, and left (M1) and right (M2).

When preprocessing and measurements of EEG data, researchers were blind concerning subjects' identity and diagnosis, but acquisition of EEG signals was not blinded.

\section{Statistical Analysis}

Quantitative data are presented as the mean \pm standard deviation (SD). The comparisons of two groups in continuous demographics and clinical variables were analyzed by independent $t$-tests or the $\chi^{2}$ test. Repeated-measures analysis of variance (ANOVA) was used to analyze the EMMN mean amplitude, with expression (happy and sad), electrode (P7/P8, O1/O2 and M1/M2) and hemisphere (left and right) as within-subject factors, and group (OSAS patients vs healthy controls) as a between-subject factor. The degrees of freedom were corrected using GreenhouseGeisser epsilon for a sphericity assumption violation. Further post hoc analysis was carried out using the Bonferroni correction. A one tailed $t$-test was conducted to reflect differences in the EMMN mean amplitude and zero as described by Yin et al. ${ }^{27}$ Pearson product-moment correlation coefficients (r) were calculated to discuss the correlation between neuropsychological test scores, clinical variables, and electrophysiological data. All analyses were performed using SPSS 23.0 software (SPSS Inc., Chicago, IL, USA) and results with $P<0.05$ were considered as statistically significant. Effect sizes of results were reported as partial eta squared $\left(\eta^{2}\right)$.

\section{Results}

\section{Subject Characteristics}

The demographic and PSG data of healthy controls and patients with OSAS are shown in Table 1. There were no significant differences in age, gender, and education level between two groups (all $P>0.1$ ). However, OSAS patients presented significantly greater BMI than control subjects $(P<0.001)$. Patients with OSAS had an illness history ranging from 4 to 5 years. Moreover, between

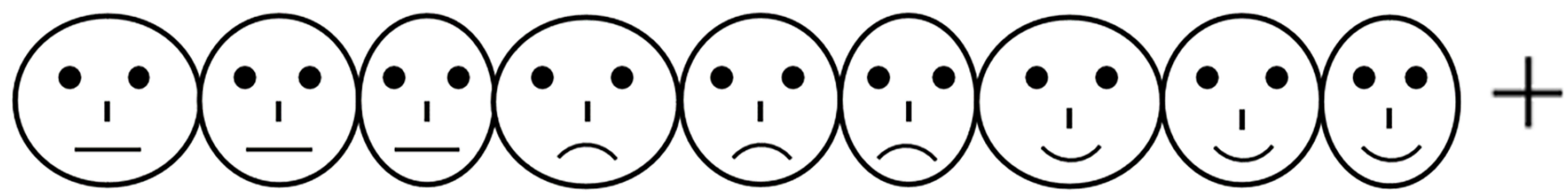

Figure I Samples of the fixation cross and standard (neutral faces) and deviant stimuli (happy or sad faces) that were mediated by altering the shape and distance between the facial features. 


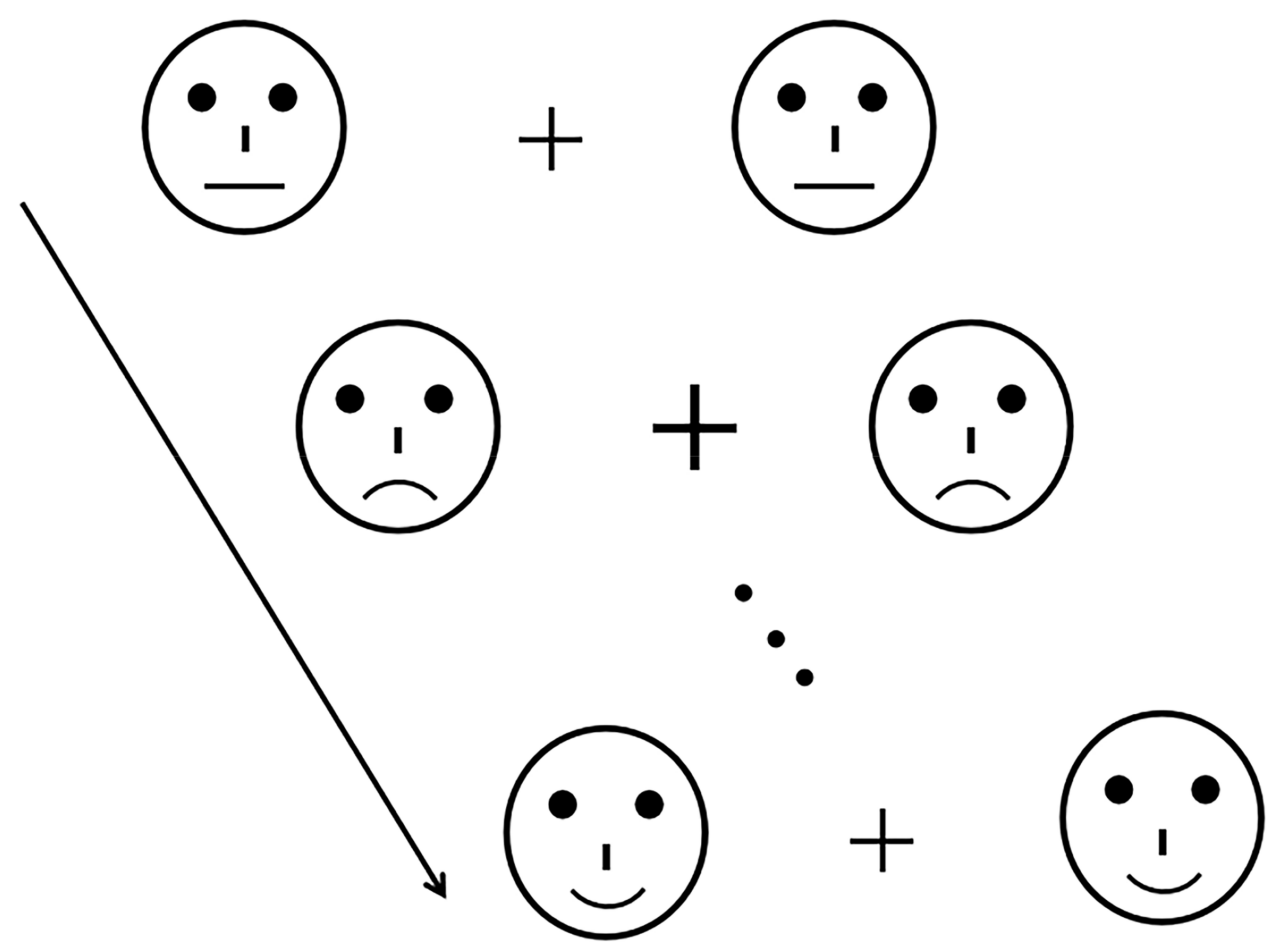

Figure 2 The expression-related oddball paradigm.

groups, greater differences were observed for sleep parameters, which included AHI, ODI, $\mathrm{MSpO}_{2}$, and $\mathrm{MmSpO}_{2}$ \% $(P<0.001, P<0.001, P<0.001$ and $P=0.001$, respectively). In terms of daytime sleepiness, assessed by ESS, OSAS patients were clearly higher score compared with healthy controls $(P<0.001)$, and there was lower score in MOCA scores for OSAS groups $(P<0.001)$.

\section{Behavioral Performance}

The participants' reaction time (RT) to the cross changes was evaluated and there were no significant differences between OSAS patients $(362.22 \pm 32.87 \mathrm{~ms})$ and healthy controls $(355.84 \pm 39.79 \mathrm{~ms}, P=0.569)$.

\section{ERP results}

The grand-averaged ERP waveforms elicited by standard (neutral faces) and deviant stimuli (happy and sad faces) in OSAS patients and $\mathrm{HC}$ at $\mathrm{P} 7 / \mathrm{P} 8$ sites are presented in Figure 3, while the grand-averaged EMMN waveforms are shown in Figure 4 and the analyses of EMMN components are summarized in Table 2 .

In early time window of $150-250 \mathrm{~ms}$, there was a significant main effect of group $(\mathrm{F}(1,41)=14.895$, $P<0.001$, partial $\left.\eta^{2}=0.266\right)$, indicating larger early EMMN amplitudes (more negative) in controls $(-0.55 \pm$
$0.13 \mu \mathrm{V})$ compared with OSAS sufferers $(0.18 \pm 0.13 \mu \mathrm{V})$. Across groups, facial expressions were shown to modulate EMMN amplitude $\left(\mathrm{F}(1,41)=9.501, P=0.004\right.$, partial $\eta^{2}$ $=0.188)$, and the interaction between group and emotion was significant $\left(\mathrm{F}(1,41)=5.095, P=0.029\right.$, partial $\eta^{2}=$ 0.111). Post-hoc analysis showed that both amplitudes of happy EMMN and sad EMMN in HC group $(-0.26 \pm 0.16$ $\mu \mathrm{V}$ for happy EMMN, $-0.83 \pm 0.14 \mu \mathrm{V}$ for sad EMMN) were larger (more negative) than those of the OSAS group $(0.22 \pm 0.16 \mu \mathrm{V}, P=0.04$ for happy EMMN; $0.13 \pm 0.14$ $\mu \mathrm{V}, P<0.001$ for sad EMMN), implying that amplitudes of EMMN in patients with OSAS were significantly decreased, regardless of happy or sad condition. Furthermore, EMMN amplitude elicited by sad facial expression $(-0.83 \pm 0.15 \mu \mathrm{V})$ was larger than that of happy facial expression $(-0.26 \pm 0.16 \mu \mathrm{V})$ in $\mathrm{HC}$ group $(P=0.001)$, but this phenomenon was not observed in OSAS group $(P=0.558)$. Additionally, we found noticeable main effect of electrode $(\mathrm{F}(1.39,56.80)=5.78, P=$ 0.012 , partial $\left.\eta^{2}=0.123\right)$ as well, with most negative EMMN amplitude at P7/P 8 electrodes $(-0.36 \pm 0.09 \mu \mathrm{V})$.

Similar to results in early EMMN analysis, the late EMMN amplitude was significantly reduced in OSAS patients $(0.31 \pm 0.15 \mu \mathrm{V})$ compared with $\mathrm{HC}$ group ($0.57 \pm 0.15 \mu \mathrm{V}, \mathrm{F}(1,41)=17.610, P<0.001$, partial $\eta^{2}$ 

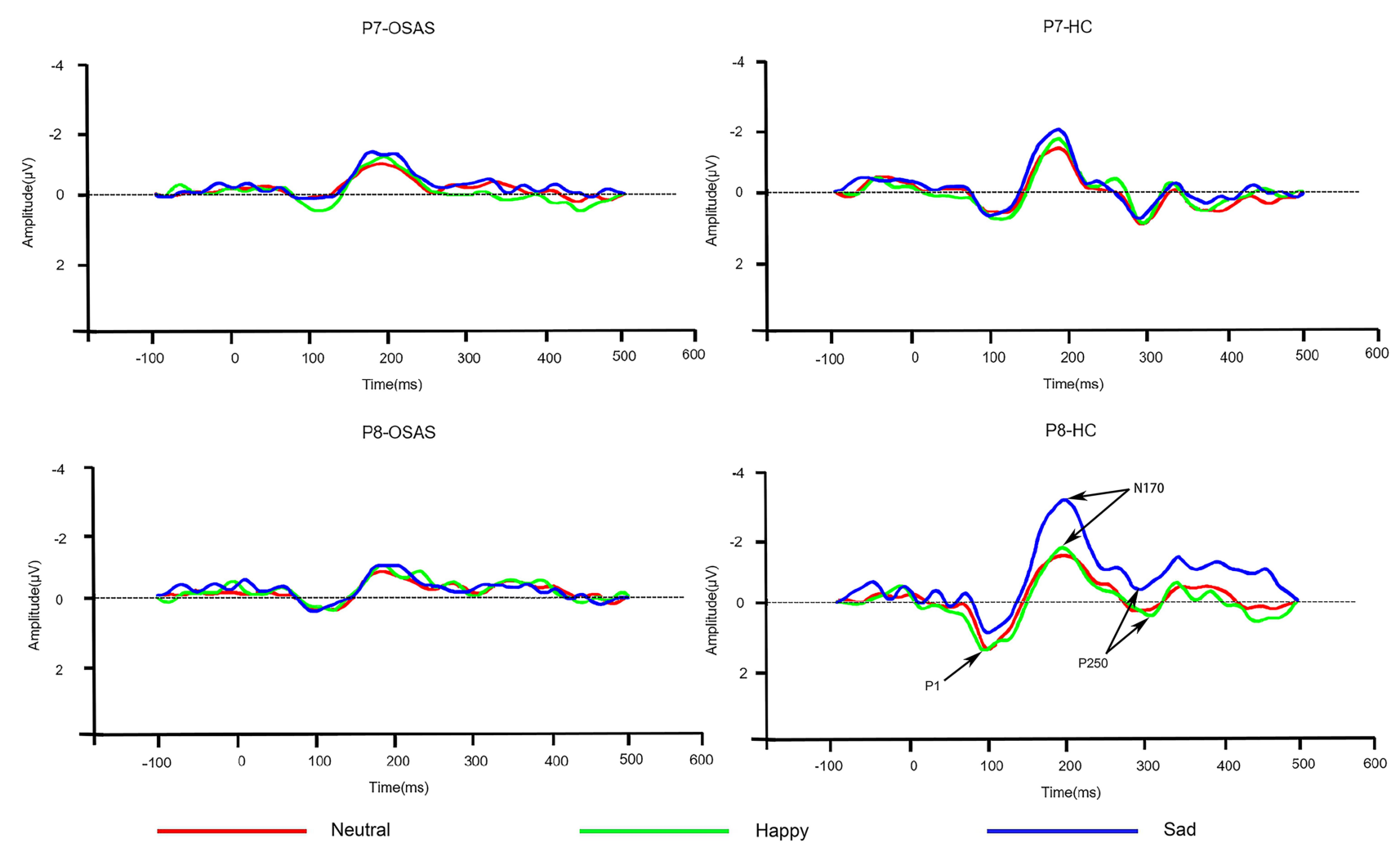

Figure 3 Grand-averaged ERP waveforms for standard (neutral faces) and deviant stimuli (happy and sad faces) at P7 and P8 sites.

$=0.300)$. The expression effect $(\mathrm{F}(1,41)=9.658, P<$ 0.003 , partial $\eta^{2}=0.191$ ), together with a significant group $\times$ expression interaction $(\mathrm{F}(1,41)=4.552, P=0.039$, partial $\left.\eta^{2}=0.100\right)$, were obtained as well. Subsequent comparisons demonstrated that significant differences existed under both happy condition $(0.36 \pm 0.18 \mu \mathrm{V}$ and $0.29 \pm 0.18 \mu \mathrm{V}$ for OSAS patients and $\mathrm{HC}$, respectively; $P=0.014)$ and sad condition $(0.26 \pm 0.15 \mu \mathrm{V}$ and $-0.86 \pm$ $0.16 \mu \mathrm{V}$ for OSAS patients and $\mathrm{HC}$, respectively; $P<$ 0.001). In HC group, sad EMMN $(-0.86 \pm 0.16 \mu \mathrm{V})$ was found to be larger than happy EMMN $(0.29 \pm 0.18 \mu \mathrm{V}, P=$ $0.001)$, but not in patients with OSAS $(P=0.490)$.

In order to further demonstrate the existence of EMMN, the EMMN amplitude of each group was compared to zero. For early and late EMMN of HC group, the mean amplitude of EMMN at each channel was significantly different from zero $(P \mathrm{~s}<0.01)$, regardless of happy or sad faces, except for the mean amplitude of EMMN elicited by happy facial expressions at the M1/M2 channel (all $P>0.05$ ). On the contrary, in the OSAS group, these comparisons did not reach significant levels for both happy EMMN and sad EMMN (all $P>0.05$ ), indicating the absence of early EMMN, regardless of happy or sad conditions.

\section{Correlations Between EMMN Amplitude and Clinical Characteristics}

We analyzed the correlations between EMMN amplitude and Age, BMI, AHI, ODI, $\mathrm{MSpO}_{2}, \mathrm{MmSpO}_{2} \%$, AI, MOCA and ESS in OSAS patients and healthy controls (Table 3). The amplitude of EMMN was positively correlated with AHI, BMI and ESS, indicating that more apneahypopnea events were associated with more negative EMMN amplitudes. EMMN amplitude also showed significant and negative correlations with $\mathrm{MSpO}_{2}$ and MOCA, whether under happy or sad conditions. However, ODI was only positively correlated with early and late happy EMMN. No obvious correlations were found between amplitude of EMMN and clinical characteristics in healthy controls.

\section{Discussion}

In the present study, using an expression-related oddball paradigm to investigate pre-attentive processing of facial expressions, we found that although the reaction time for detecting cross change did not differ between two groups, the amplitude of EMMN declined significantly in patients with OSAS compared with HC, regardless of sad or happy 
Table I Demographic and Clinical Characteristics of Healthy Controls and OSAS Patients

\begin{tabular}{|l|l|l|l|l|}
\hline & $\begin{array}{l}\text { Healthy } \\
\text { Controls }\end{array}$ & $\begin{array}{l}\text { OSAS } \\
\text { Patients }\end{array}$ & $\mathbf{t} / \chi 2$ & $\mathbf{P}$ \\
\hline $\mathrm{N}$ & 21 & 22 & & \\
\hline Age & $42.7 \mathrm{I} \pm 5.85$ & $44.59 \pm 4.75$ & -1.158 & 0.254 \\
\hline Age range & $30-53$ & $35-56$ & & \\
\hline Gender (M/F) & $11 / 10$ & $14 / 8$ & 0.559 & 0.455 \\
\hline Education(year) & $12.29 \pm 2.05$ & $12.27 \pm 2.39$ & 0.019 & 0.985 \\
\hline BMI (kg/m $\left.{ }^{2}\right)$ & $25.66 \pm 1.61$ & $28.57 \pm 1.34$ & -6.474 & $0.000^{* * *}$ \\
\hline History (year) & - & $4.23 \pm 0.922$ & & \\
\hline AHI(events/h) & $2.62 \pm 1.02$ & $23.59 \pm 4.02$ & -23.664 & $0.000^{* * *}$ \\
\hline ODI & $6.62 \pm 2.92$ & $27.82 \pm 4.86$ & -17.238 & $0.000^{* * *}$ \\
\hline MSpO2 & $96.52 \pm 0.93$ & $94.55 \pm 1.26$ & 5.832 & $0.000^{* * *}$ \\
\hline MmSpO2\% & $85.90 \pm 3.42$ & $81.23 \pm 4.64$ & 3.749 & $0.001 * *$ \\
\hline Al & $19.38 \pm 5.49$ & $21.18 \pm 4.67$ & -1.161 & 0.252 \\
\hline MoCA & $27.33 \pm 0.91$ & $25.41 \pm 1.37$ & 5.447 & $0.000^{* * *}$ \\
\hline ESS & $4.95 \pm 2.58$ & $17.82 \pm 2.54$ & -16.488 & $0.000^{* * * *}$ \\
\hline Notes Da were exp & & & & \\
\hline
\end{tabular}

Notes: Data were expressed as mean $\pm \mathrm{SD}$. **P $<0.01$, ***P $<0.00$ I by Student's $t$-test (two-tailed).

Abbreviations: OSAS, obstructive sleep apnea syndrome; M, male; F, female; BMI, body mass index; ESS, Epworth Sleepiness Scale; AHI, apnea hypopnea index; ODI, oxygen desaturation index; $\mathrm{MSpO} 2$, mean oxygen saturation during the night; $\mathrm{MmSpO} 2 \%$, lowest oxygen saturation during the night; $\mathrm{Al}$, arousal index; MoCA, Montreal Cognitive Assessment.

condition. In addition, these electrophysiological abnormalities were directly correlated with $\mathrm{AHI}$ and $\mathrm{MSpO}$.

In our study, we found that the significant group effects of EMMN mean amplitudes, and post hoc analyses showed that the amplitudes of EMMN were significantly reduced in OSAS patients compared with healthy control, regardless of happy or sad condition. Since MMN could be elicited even in the absence of attention or behavioral response and reflect the capability of automatic processing of sensory information, ${ }^{34,35}$ the aforementioned results suggested that patients with OSAS might suffer from preattentive emotional processing abnormalities. Furthermore, source localization analysis was performed by Kimura et $\mathrm{al}^{34}$ further demonstrating that EMMN had its main generators in frontal, limbic, temporal and occipital lobes, and the activation of occipitotemporal lobe was observed in early EMMN, while frontal and limbic activations occurred in almost all stages of EMMN. Interestingly, Yaouhi et al confirmed gray matter loss in the temporo- parieto-occipital and frontal cortices using a highresolution MRI scanning in OSAS patients. ${ }^{36}$ Recent study of cerebral functional imaging and damage showed that decreased brain gray matter volume with quantitative neuroimaging in OSAS patients. ${ }^{37}$ Thus, there is the possibility that alterations in corresponding brain regions might explain pre-attentive dysfunction of facial expressions, while the underlying neural mechanisms deserve further elucidation.

Another key finding was that the mean amplitude of EMMN for sad facial expressions was significantly larger than that for happy facial expressions in the control group, implying that healthy people are more sensitive and able to process negative emotional information. In line with our findings, previous studies have shown that EMMN reflected pre-attentive change detection of facial emotion, with larger amplitude for negative facial expression than for positive one in controls. ${ }^{23,38}$ In addition, a study proposed that people could process negative emotional information more quickly and efficiently. ${ }^{39}$ From an electrophysiological perspective, the abovementioned results supported the proposed theory of negative bias effect.

In order to assess the relationships between EMMN amplitudes and clinical variables of OSAS sufferers, Pearson's correlations were calculated. Almost no correlations were found between EMMN and Age, AI, $\mathrm{MmSpO}_{2}$ $\%$, but we discovered that there were positive correlations between EMMN amplitudes and BMI, ESS, AHI, and they were also negatively correlated with $\mathrm{MSpO}_{2}$, indicating that several clinical characteristics of OSAS patients could potentially modulate pre-attentive emotion processing abnormalities. Consistent with our results, some studies found that sleepiness cause attenuation amplitude of auditory MMN, these results could suggest that cognitive pre-attentive processing is impaired. ${ }^{40,41}$ Furthermore, an interesting result is that the amplitude of EMMN was negatively correlated with MoCA score. Montreal Cognitive Assessment (MoCA) covering various important domains of neurocognitive functions, ${ }^{31}$ which was used to assess eight aspects of cognitive functions, these eight aspects were subdivided into 12 themes and 30 separate items. Although abovementioned brain function field could not enough to explain the cognitive impairment of automatic processing of emotional facial expressions under the pre-attentive condition in patients with OSAS, one possible explanation is that EMMN reflects mismatches between an infrequent facial expression and the 

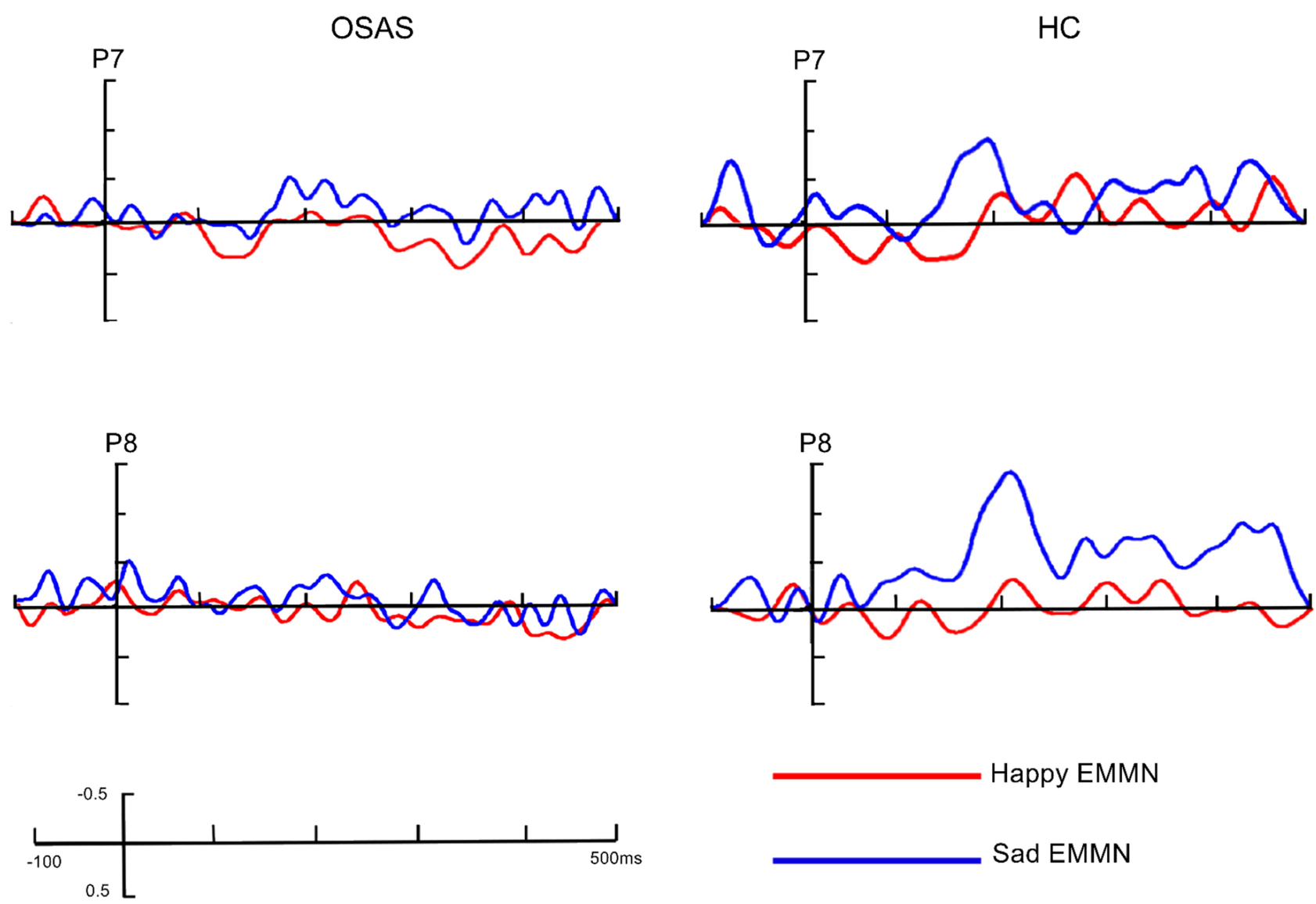

Happy EMMN

Sad EMMN

Figure 4 The grand-averaged EMMN waveforms in patients with OSAS and healthy controls at P7/P8 site.

memory of a frequent facial expression, and is likely to represent a wider range of neurocognitive associations. ${ }^{35}$ Future research results may further promote understanding of the neuropsychological mechanism of emotion cognition and increase the brain's understanding of this part of emotional facial expressions under the pre-attentive condition processing function.

The main advantages of the present study are standardized subject selection criteria and collection of detailed OSAS characteristics. In addition, this is the first research to examine relations between expression-related MMN and OSAS by using expression-related oddball paradigm with high sensitivity. Previous findings have indicated that simple schematic faces might be beneficial to clinical research and application, ${ }^{25}$ so multiple schematic facial models were used to avoid low-level processing of facial features and eliminate the possible influence of facial gender and race. It should be noted that this study has several limitations constraining explanation of our present findings. Firstly, the small sample size might have confined the study of the effects of gender, age and

Table 2 Results and Analyses of Mean EMMN Amplitudes $(\mu \mathrm{V})$ in Healthy Controls and OSAS Patients

\begin{tabular}{|c|c|c|c|c|c|c|c|}
\hline \multirow[t]{2}{*}{ Time } & \multicolumn{2}{|c|}{$\begin{array}{l}\text { Healthy Controls EMMN } \\
\text { Amplitude }(\mu \mathrm{V})\end{array}$} & \multicolumn{2}{|c|}{$\begin{array}{l}\text { OSAS Patients EMMN } \\
\text { Amplitude }(\mu \mathrm{V})\end{array}$} & \multicolumn{3}{|l|}{ Statistics } \\
\hline & Happy & Sad & Happy & Sad & $\begin{array}{l}\text { Group } \\
F(I, 4 I) / \eta 2\end{array}$ & $\begin{array}{l}\text { Emotion } \\
F(I, 4 I) / \eta 2\end{array}$ & $\begin{array}{l}\text { Group } \times \text { Emotion } \\
F(I, 4 I) / n 2\end{array}$ \\
\hline $150-250 \mathrm{~ms}$ & $-0.26 I \pm 0.163$ & $-0.830 \pm 0.145$ & $0.224 \pm 0.160$ & $0.136 \pm 0.142$ & $14.895 * * * / 0.266$ & $9.501 * * / 0.188$ & $5.095 * / 0.111$ \\
\hline $250-350 \mathrm{~ms}$ & $-0.290 \pm 0.183$ & $-0.855 \pm 0.155$ & $0.360 \pm 0.178$ & $0.260 \pm 0.152$ & $17.610^{* * * *} / 0.300$ & $9.658 * * / 0.191$ & $4.552 * / 0.100$ \\
\hline
\end{tabular}

Notes: Data were expressed as mean $\pm \mathrm{SD}$. $* \mathrm{P}<0.05,{ }^{*} * \mathrm{P}<0.01 * * * \mathrm{P}<0.001$ by analysis of variance with repeated measures (Bonferroni correction). 
Table 3 Correlations Between Mean EMMN Amplitudes and Clinical Characteristics in OSAS Patients and Healthy Controls

\begin{tabular}{|c|c|c|c|c|}
\hline \multirow{2}{*}{$\begin{array}{l}\text { Group } \\
\text { OSAS patients }\end{array}$} & \multicolumn{2}{|c|}{$\begin{array}{l}\text { Early EMMN Amplitude } \\
(150-250 \mathrm{~ms})\end{array}$} & \multicolumn{2}{|c|}{$\begin{array}{l}\text { Late EMMN Amplitude } \\
(250-350 \mathrm{~ms})\end{array}$} \\
\hline & $\begin{array}{l}\text { Happy EMMN } \\
r \text { ( } p \text { value })\end{array}$ & $\begin{array}{l}\text { Sad EMMN } \\
r \text { ( } p \text { value) }\end{array}$ & $\begin{array}{l}\text { Happy EMMN } \\
r \text { ( } p \text { value })\end{array}$ & $\begin{array}{l}\text { Sad EMMN } \\
r \text { ( } p \text { value) }\end{array}$ \\
\hline Age & $0.048(0.024)^{*}$ & $-0.021(0.925)$ & $0.359(0.101)$ & $0.207(0.355)$ \\
\hline BMI & $0.548(0.008)^{* *}$ & $0.662(0.001)^{* *}$ & $0.496(0.019)^{*}$ & $0.502(0.017)^{*}$ \\
\hline $\mathrm{AHI}$ & $0.536(0.015)^{*}$ & $0.516(0.020)^{*}$ & $0.460(0.04 \mathrm{I})^{*}$ & $0.606(0.005)^{* *}$ \\
\hline ODI & $0.529(0.017)^{*}$ & $0.198(0.404)$ & $0.444(0.050)^{*}$ & $0.268(0.253)$ \\
\hline $\mathrm{MSpO} 2$ & $-0.495(0.026)^{*}$ & $-0.464(0.039)^{*}$ & $-0.483(0.03 \mathrm{I})^{*}$ & $-0.363(0.003)^{* *}$ \\
\hline $\mathrm{MmSpO} \%$ & $-0.361(0.118)$ & $0.076(0.750)$ & $-0.317(0.173)$ & $-0.149(0.532)$ \\
\hline $\mathrm{Al}$ & $-0.125(0.600)$ & $-0.380(0.099)$ & $-0.126(0.598)$ & $-0.329(0.156)$ \\
\hline MOCA & $-0.662(0.001)^{* *}$ & $-0.423(0.050)^{*}$ & $-0.607(0.003)^{* *}$ & $-0.558(0.007)^{* *}$ \\
\hline ESS score & $0.619(0.002)^{* *}$ & $0.477(0.025)^{*}$ & $0.579(0.005)^{* *}$ & $0.579(0.005)^{* *}$ \\
\hline Healthy controls & $\begin{array}{l}\text { Happy EMMN } \\
r \text { ( } p \text { value })\end{array}$ & $\begin{array}{l}\text { Sad EMMN } \\
r \text { ( } p \text { value) }\end{array}$ & $\begin{array}{l}\text { Happy EMMN } \\
r \text { ( } p \text { value) }\end{array}$ & $\begin{array}{l}\text { Sad EMMN } \\
r \text { ( } p \text { value) }\end{array}$ \\
\hline Age & $0.088(0.593)$ & $0.064(0.607)$ & $0.059(0.693)$ & $0.147(0.586)$ \\
\hline BMI & $0.103(0.635)$ & $0.198(0.401)$ & $0.116(0.613)$ & $0.216(0.321)$ \\
\hline $\mathrm{AHI}$ & $0.321(0.136)$ & $0.317(0.172)$ & $0.362(0.114)$ & $0.308(0.179)$ \\
\hline ODI & $0.184(0.425)$ & $-0.013(0.94 I)$ & $0.151(0.489)$ & $0.079(0.700)$ \\
\hline $\mathrm{MSpO} 2$ & $0.283(0.135)$ & $0.249(0.210)$ & $0.314(0.052)$ & $0.225(0.250)$ \\
\hline $\mathrm{MmSpO} 2 \%$ & $0.161(0.493)$ & $-0.159(0.510)$ & $0.124(0.601)$ & $0.096(0.698)$ \\
\hline $\mathrm{Al}$ & $0.157(0.488)$ & $-0.083(0.716)$ & $0.327(0.135)$ & $0.175(0.437)$ \\
\hline MOCA & $0.032(0.878)$ & $0.001(0.997)$ & $0.046(0.0853)$ & $0.056(0.803)$ \\
\hline ESS score & $0.115(0.616)$ & $0.200(0.398)$ & $0.126(0.598)$ & $0.199(0.400)$ \\
\hline
\end{tabular}

Notes: Early EMMN in the windows of $150-250 \mathrm{~ms}$, Late EMMN in the windows of $250-350 \mathrm{~ms}$. $r$ represents product-moment correlation coefficient. *P < 0.05 , **P < 0.01 by Pearson's correlations (two-tailed).

specific clinical characteristics, and the distinctions between the severity of OSAS among patients were not explored either. Larger cohorts should be increased to explore the differences of EMMN amplitude in mild, moderate and severe patients and regression analysis should be added to explore the influence of relevant factors on EMMN amplitude. Secondly, although schematic emotional faces were simple and reliable, ${ }^{42}$ real faces should also be used to further investigate this issue. Finally, source analysis was not conducted in this experiment, and we wish to work on it in future studies to reveal the underlying areas of network-level pathology.

\section{Conclusions}

Our study revealed pre-attentive processing of task-irrelevant emotional information was impaired in patients with OSAS, regardless of sad or happy facial expressions, meanwhile the negative bias effect was also not found, suggesting that the advantage of OSAS patients in automatic processing of sad expression was not obvious compared with that of happy expression. Notably, these electrophysiological parameters were negatively correlated with $\mathrm{MSpO}_{2}$ and positive correlations with AHI, indicating the potential effect of some clinical characteristics of OSAS patients on automatic emotional processing. Considering the high prevalence and neurocognitive impairment of OSAS, EMMN might be used as a sensitive electrophysiological biomarker, while the reliability and efficacy demand further investigations.

\section{Disclosure}

The authors report no potential conflicts of interest for this work.

\section{References}

1. Zhang X, Ma L, Li S, et al. A functional MRI evaluation of frontal dysfunction in patients with severe obstructive sleep apnea. Sleep Med. 2011;12(4):335-340.

2. Beebe DW, Gozal D. Obstructive sleep apnea and the prefrontal cortex: towards a comprehensive model linking nocturnal upper airway obstruction to daytime cognitive and behavioral deficits. J Sleep Res. 2002;11(1):1-16.

3. Gagnon K, Baril AA, Gagnon JF, et al. Cognitive impairment in obstructive sleep apnea. Pathol Biol (Paris). 2014;62(5):233-240. 
4. Jackson ML, Howard ME, Barnes M. Cognition and daytime functioning in sleep-related breathing disorders. Prog Brain Res. 2011;190:53-68.

5. Peppard PE, Young T, Barnet JH, Palta M, Hagen EW, Hla KM. Increased prevalence of sleep-disordered breathing in adults. $\mathrm{Am}$ J Epidemiol. 2013;177(9):1006-1014.

6. Osorio RS, Gumb T, Pirraglia E, et al. Sleep-disordered breathing advances cognitive decline in the elderly. Neurology. 2015;84 (19):1964-1971.

7. Gosselin N, Mathieu A, Mazza S, Petit D, Malo J, Montplaisir J. Attentional deficits in patients with obstructive sleep apnea syndrome: an event-related potential study. Clin Neurophysiol. 2006;117(10):2228-2235.

8. Adams N, Strauss M, Schluchter M, Redline S. Relation of measures of sleep-disordered breathing to neuropsychological functioning. $\mathrm{Am}$ J Respir Crit Care Med. 2001;163(7):1626-1631.

9. Grenèche J, Krieger J, Bertrand F, Erhardt C, Maumy M, Tassi P. Short-term memory performances during sustained wakefulness in patients with obstructive sleep apnea-hypopnea syndrome. Brain Cogn. 2011;75(1):39-50.

10. Turner K, Zambrelli E, Lavolpe S, Baldi C, Furia F, Canevini MP. Obstructive sleep apnea: neurocognitive and behavioral functions before and after treatment. Funct Neurol. 2019;34(2):71-78.

11. Näätänen R, Kujala T, Kreegipuu K, et al. The mismatch negativity: an index of cognitive decline in neuropsychiatric and neurological diseases and in ageing. Brain. 2011;134(Pt 12):3435-3453.

12. Gelir E, Başaran C, Bayrak S, et al. Electrophysiological assessment of the effects of obstructive sleep apnea on cognition. PLoS One. 2014;9(2):e90647.

13. Näätänen $R$, Kujala $T$, Escera $C$, et al. The mismatch negativity (MMN)-a unique window to disturbed central auditory processing in ageing and different clinical conditions. Clin Neurophysiol. 2012;123(3):424-458.

14. Näätänen R, Pakarinen S, Rinne T, Takegata R. The mismatch negativity (MMN): towards the optimal paradigm. Clin Neurophysiol. 2004;115(1):140-144.

15. Wen X, Wang N, Liu J, Yan Z, Xin Z. Detection of cognitive impairment in patients with obstructive sleep apnea hypopnea syndrome using mismatch negativity. Neural Regen Res. 2012;7(20):1591-1598.

16. Zou K, Sun Y, Tang X, et al. Sheng Wu Yi Xue Gong Cheng Xue Za Zhi. J Biomed Eng. 2014;31(4):870-874.

17. Czigler I. Visual mismatch negativity: violation of nonattended environmental regularities[J]. J Psychophysiol. 2007;21(21):224-230.

18. Pazo-Alvarez P, Amenedo E, Lorenzo-López L, Cadaveira F. Effects of stimulus location on automatic detection of changes in motion direction in the human brain. Neurosci Lett. 2004;371(2-3):111-116.

19. Czigler I, Balázs L, Pató LG. Visual change detection: event-related potentials are dependent on stimulus location in humans. Neurosci Lett. 2004;364(3):149-153.

20. Sulykos I, Czigler I. One plus one is less than two: visual features elicit non-additive mismatch-related brain activity. Brain Res. 2011;1398:64-71.

21. Czigler I. Visual mismatch negativity and categorization. Brain Topogr. 2014;27(4):590-598.

22. Susac A, Ilmoniemi RJ, Pihko E, Supek S. Neurodynamic studies on emotional and inverted faces in an oddball paradigm. Brain Topogr. 2004;16(4):265-268

23. Zhao L, Li J. Visual mismatch negativity elicited by facial expressions under non-attentional condition. Neurosci Lett. 2006;410 (2):126-131
24. Astikainen P, Hietanen JK. Event-related potentials to task-irrelevant changes in facial expressions. Behav Brain Funct. 2009;5:30.

25. Wright CI, Martis B, Shin LM, Fischer H, Rauch SL. Enhanced amygdala responses to emotional versus neutral schematic facial expressions. Neuroreport. 2002;13(6):785-790.

26. Wu Z, Zhong X, Peng Q, Chen B, Mai N, Ning Y. Negative bias in expression-related mismatch negativity (MMN) in remitted late-life depression: an event-related potential study. $J$ Psychiatr Res. 2017;95:224-230.

27. Yin G, She S, Zhao L, Zheng Y. The dysfunction of processing emotional faces in schizophrenia revealed by expression-related visual mismatch negativity. Neuroreport. 2018;29(10):814-818.

28. Zhang Y, Chen J, Hou X, et al. Dysfunction of processing task-irrelevant emotional faces in primary insomnia patients: an evidence from expression-related visual MMN. Sleep Breath. 2020. doi:10.1007/s11325-020-02058-5

29. Guo Y, Chen J, Hou X, et al. Pre-attentive dysfunction of processing emotional faces in interictal migraine revealed by expression-related visual mismatch negativity. Brain Res. 2020;1738:146816.

30. Kapur VK, Auckley DH, Chowdhuri S, et al. Clinical practice guideline for diagnostic testing for adult obstructive sleep apnea: an American academy of sleep medicine clinical practice guideline. J Clin Sleep Med. 2017;13(3):479-504.

31. Nasreddine ZS, Phillips NA, Bédirian V, et al. The Montreal cognitive assessment, MoCA: a brief screening tool for mild cognitive impairment. J Am Geriatr Soc. 2005;53(4):695-699.

32. Johns MW. A new method for measuring daytime sleepiness: epworth sleepiness scale. Sleep. 1991;14(6):540-545.

33. Jung TP, Makeig S, Humphries C, et al. Removing electroencephalographic artifacts by blind source separation. Psychophysiology. 2000;37(2):163-178.

34. Kimura M, Kondo H, Ohira H, Schröger E. Unintentional temporal context-based prediction of emotional faces: an electrophysiological study. Cereb Cortex. 2012;22(8):1774-1785.

35. Kimura M, Schröger E, Czigler I. Visual mismatch negativity and its importance in visual cognitive sciences. Neuroreport. 2011;22 (14):669-673.

36. Yaouhi K, Bertran F, Clochon P, et al. A combined neuropsychological and brain imaging study of obstructive sleep apnea. J Sleep Res. 2009;18(1):36-48

37. Baima CB, Fim NC, Alves KF, Resende LAL, Fonseca RG, Betting LE. Analysis of patients with obstructive sleep apnea with and without pharyngeal myopathy using brain neuroimaging. Sleep. 2020;43(2):zsz216.

38. Stefanics G, Csukly G, Komlósi S, Czobor P, Czigler I. Processing of unattended facial emotions: a visual mismatch negativity study. Neuroimage. 2012;59(3):3042-3049.

39. Fox E, Lester V, Russo R, Bowles RJ, Pichler A, Dutton K. Facial expressions of emotion: are angry faces detected more efficiently? Cogn Emot. 2000;14(1):61-92.

40. Sallinen M, Lyytinen H. Mismatch negativity during objective and subjective sleepiness. Psychophysiology. 1997;34(6):694-702.

41. Gumenyuk V, Roth T, Korzyukov O, et al. Shift work sleep disorder is associated with an attenuated brain response of sensory memory and an increased brain response to novelty: an ERP study. Sleep. 2010;33(5):703-713.

42. Kreegipuu K, Kuldkepp N, Sibolt O, Toom M, Allik J, Näätänen R. vMMN for schematic faces: automatic detection of change in emotional expression. Front Hum Neurosci. 2013;7:714. 
Nature and Science of Sleep

Dovepress

\section{Publish your work in this journal}

Nature and Science of Sleep is an international, peer-reviewed, open access journal covering all aspects of sleep science and sleep medicine, including the neurophysiology and functions of sleep, the genetics of sleep, sleep and society, biological rhythms, dreaming, sleep disorders and therapy, and strategies to optimize healthy sleep.
The manuscript management system is completely online and includes a very quick and fair peer-review system, which is all easy to use. Visit http://www.dovepress.com/testimonials.php to read real quotes from published authors.

Submit your manuscript here: https://www.dovepress.com/nature-and-science-of-sleep-journal 